\title{
Cardiovascular toxicities of systemic treatments of prostate cancer: oestrogen to the rescue?
}

\author{
Syed Imran A. Shah, Hannah C. P. Wilson \& Paul D. Abel
}

In their Review (Cardiovascular toxicities of systemic treatments of prostate cancer. Nat. Rev. Urol. 14, 230-243 (2017) $)^{1}$ Veccia et al. offer useful insight into current knowledge concerning cardiovascular complications of oral oestrogen, androgen deprivation therapy (ADT), and prostate cancer. However, we do not share the confidence and strength of their assertion that "oestrogens are no longer used in patients with prostate cancer owing to the severity of their adverse events, which include thromboembolic and cardiovascular effects". This misconception, based on the oral mode of administration of the drug, has led to a long period of disuse, but early data derived from the current UK national Prostate Adenocarcinoma TransCutaneous Hormones (PATCH) study $^{2,3}$, promise to improve our understanding of the value of oestrogen for treating prostate cancer and to explain the reasons for the various adverse effects (including, for example, osteoporosis and metabolic syndrome). This study could also provide a possible route to personalized medicine in hormone therapy for prostate cancer and an improved quality of life for men with this disease.

Initially, ADT (or total orchidectomy) was widely accepted to achieve androgen suppression for treating advanced prostate cancer. However, the Veterans' Administration Cooperative Urological Research Group studies on prostate cancer revealed unexpected outcomes; oral oestrogen resulted in improved prostatecancer-specific survival, but overall survival worsened substantially, exposing patients to particularly serious cardiovascular and thromboembolic toxicities arising from oral use $^{4}$. Thus, oral oestrogen, which necessarily passes through the enterohepatic circulation and bathes the liver in high levels of oestrogen resulting in induction of procoagulant molecules ${ }^{5}$, was soon abandoned ${ }^{4}$.
By the early 1990s, reports of parenteral oestrogen administration for prostate cancer (injection or skin patch) had been encouraging. A Scandinavian research team recruited 915 men into a two-arm study of luteinising-hormone-releasing hormone (LHRH) agonist plus antiandrogens versus intramuscular polyestradiol phosphate (a synthetic oestrogen). Overall prostate cancer mortality was equivalent between groups, but cardiovascular morbidity was either not reported or slightly increased in the oestrogen $\operatorname{arm}^{6}$. Application of transdermal oestrogen patches (used for hormonereplacement therapy in women) in a phase II, single-arm study of 20 men with prostate cancer reported just one case of cardiovascular toxicity at 12-months follow-up duration ${ }^{7}$. Most recently, the ongoing PATCH trial has now recruited $>1,300$ men into two study arms, LHRH agonist or oestrogen patches. The independent data trial monitoring committee have unlimited access to any data, and, to date, have not reported any reason to pause or stop the trial, suggesting no considerable disadvantage to transdermal oestrogen ${ }^{2}$.

The final PATCH trial data are needed for confirmation (which are estimated in 2023), but current analysis suggests that oral administration bears responsibility for the cardiovascular adverse events of oestrogen, that the consequences of oral oestrogen can be mitigated by parenteral administration.

With accumulating knowledge, it seems timely to improve investigations into the potential benefits of oestrogen therapy in prostate cancer treatment and in particular clarify the longstanding misinterpretation that oestrogen be dismissed owing to its cardiovascular toxicity. We conclude that updating knowledge of a potentially important role for oestrogen in prostate cancer management is necessary, after which patients and their clinicians will have acquired the appropriate knowledge to participate in choosing favoured therapies. Sharing decision making will offer opportunities for patients, enabling them, together with their families and friends, to engage with personalized medicine, but this process requires thorough acquisition of comprehensive, up-to-date, relevant background data.

Syed Imran A. Shah is at the Biochemistry Department at Central Park Medical College, Central Park Housing Scheme, 31 km Ferozepur Road, Kahna Nau, Lahore, Punjab, Pakistan.

Hannah C. P. Wilson is at Imperial NHS Trust, The Bays, South Wharf Road, St Mary's Hospital, London W2 $1 \mathrm{NY}$.

Paul D. Abel is at South Kensington Campus, London, SW7 2AZ, UK

Correspondence to: S.I.A.S s.shah10@alumni.imperial.ac.uk

doi:10.1038/nrurol.2017.126 Published online 8 Aug 2017

\section{Veccia, A., Maines, F., Kinspergher, S., Galligioni, E. $£$ Caffo, O. Cardiovascular toxicities of systemic treatments of prostate cancer. Nat. Rev. Urol. 14, 230-243 (2017) \\ 2. Langley, R. E. et al. Cardiovascular outcomes in patients with locally advanced and metastatic prostate cancer treated with luteinising-hormone-releasing- hormone agonists or transdermal oestrogen: the randomised, phase $2 \mathrm{MRC}$ PATCH trial (PR09). Lancet Oncol. 14, 306-316 (2013). \\ 3. Langley, R. E. et al. Early hormonal data from a multicentre phase II trial using transdermal oestrogen patches as first-line hormonal therapy in patients with locally advanced or metastatic prostate cancer. BJU Int. 102, 442-445 (2008). \\ 4. Byar, D. P. The veterans administration cooperative urological research group's studies of cancer of the prostate. Cancer 32, 1126-1130 (1973). \\ 5. Von Schoultz, B. et al. Estrogen therapy and liver function-metabolic effects of oral and parenteral administration. Prostate 14, 389-395 (1989). \\ 6. Hedlund, P. O. et al. Parenteral estrogen versus combined androgen deprivation in the treatment of metastatic prostatic cancer: part 2. Final evaluation of the Scandinavian Prostatic Cancer Group (SPCG) Study No. 5. Scand. J. Urol. Nephrol. 42, 220-229 (2008). \\ 7. Ockrim, J. L., Lalani, E. N., Laniado, M. E., Carter, S. S. \& Abel, P. D. Transdermal estradiol therapy for advanced prostate cancer-forward to the past? J. Urol. 169, 1735-1737 (2003).}

Competing interests statement

P.A. is coprincipal investigator of the UK national Prostate Adenocarcinoma TransCutaneous Hormones (PATCH) study and has grant funding from Cancer Research UK. The PATCH study is a phase III randomized controlled clinical trial comparing efficacy and toxicity of transcutaneous oestrogen patches and luteinising-hormone-releasing hormone agonist funded by Cancer Research UK and supported by the UK Medical Research council S. I. A. S. received a PhD grant from the Commonwealth Scholarship Commission. H.C.P.W. declares no competing interests.

\section{Author contributions}

S.I.A.S. and P.A. had the idea for the article, S.I.A.S researched data for the article, all authors wrote, edited and reviewed the manuscript before submission. 